\title{
OCLUSIÓN ARTERIAL AGUDA ASOCIADA A HIDATIDOSIS DISEMINADA
}

\author{
Jose Somocurcio ${ }^{1, a}$, Gamero Alvarez ${ }^{2 a}$, Gloria Lara Campos 3 ,b , Judith Cahuana Aparco ${ }^{4, c}$, \\ Estefanny Calongos Porras ${ }^{4, c}$, Pedro Bermejo Cataño ${ }^{4, c}$, Paul Tejada Llacsa ${ }^{4, c}$
}

\begin{abstract}
RESUMEN
Hidatidosis o equinococosis son términos usados para denominar a la infestación zoonótica causada por los estadios adulto y larvario (metacéstode) del céstode del género Equinococcus (familia tenidae). El término hidatidosis debe ser aplicado a la infestación por el metacéstode mientras que el de equinococosis a los estadios larvario y adulto. En el Perú existe una alta prevalencia de esta infección siendo el hígado y pulmón los órganos más afectados. Existen factores tanto del agente infeccioso como del hospedero que contribuyen a esta infección, donde el componente inmunológico del hospedero es un punto importante tanto en la infección como en la diseminación. En el presente artículo se reporta un caso de oclusión arterial aguda asociada a hidatidosis diseminada en un paciente de 54 años de edad, natural y procedente de Huancavelica, de inicio súbito con un cuadro de obstrucción arterial aguda; que no mejoró luego de la cirugía con fallecimiento a las 48 horas de la presentación.
\end{abstract}

Palabras clave: Embolia; Hidatidosis; Zoonosis (fuente: DeCS BIREME).

\section{ACUTE ARTERIAL OCCLUSION ASSOCIATED DISSEMINATED HYDATIDOSIS}

\begin{abstract}
Hydatidosis or echinococcosis are terms used to denote the zoonotic infestation caused by adult and larval stages (metacestode) of the cestode from the genus Echinococcus (family tenidae). The term hydatidosis should be applied to the infestation caused by the metacestode while echinococcosis for the larval and adult stages. In Peru there is a high prevalence of this infection, liver and lung being the most affected organs. There are factors from the host and from the infectious agent that contribute to this infection, with the immune component of the host as an important infection and dissemination point. A case of acute arterial occlusion associated with disseminated hydatidosis in a 54 years old patient, born and from Huancavelica, with sudden onset of an acute arterial obstruction is reported. The patient did not improve after surgery and died within 48 hours of presentation.
\end{abstract}

Key word: Embolism; Hydatidosis; Zoonoses (source: MeSH NLM).

\section{INTRODUCCIÓN}

La equinococosis quística, hidatidosis o enfermedad hidatídica es una infestación zoonótica causada por el estadio larvario del céstode del género Equinococcus (familia tenidae). En la actualidad se conocen por lo menos cuatro especies del género Echinococcus: Echinococcus granulosus (Batsch, 1786); Echinococcus multilocularis (Leuckart, 1863); Echinococcus oligarthrus (Diesing, 1863), y Echinococcus vogeli (Rausch y Bernstein 1972). La infección larvaria por la especie Echinococcus granulosus es la más frecuente en nuestro medio y es de especial importancia debido a su alto impacto socioeconómico y a su amplia distribución geográfica, pues ha sido reportada en más de cien países ${ }^{(1)}$, donde América del Sur es uno de los continentes más afectados (2). En el Perú, estudios epidemiológicos en poblaciones de los Andes han mostrado altas prevalencias de infección humana $(9,1 \%)^{(3)}$. Asimismo, se ha estimado un impacto negativo en la economía de este país ${ }^{(4)}$.

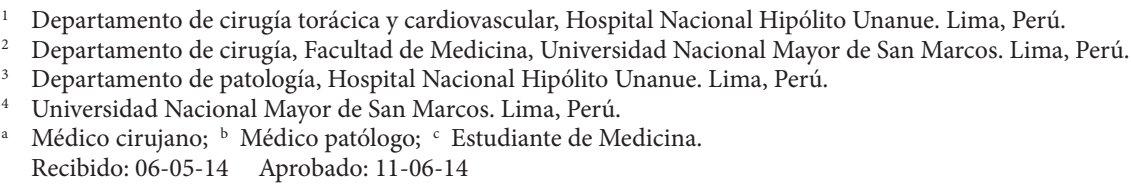

Citar como: Somocurcio J, Alvarez G, Lara Campos G, Cahuana Aparco J, Calongos Porras E, Bermejo Cataño P, et al. Oclusión arterial aguda asociada a hidatidosis diseminada. Rev Peru Med Exp Salud Publica. 2014;31(2):375-9. 
Se presenta un caso inusual de oclusión arterial aguda asociada a hidatidosis diseminada, que se manifiesta como un cuadro de embolia arterial aguda, con evolución fatal del paciente a las $72 \mathrm{~h}$ de la presentación. El paciente manifestó haber sido intervenido quirúrgicamente 8 años antes del cuadro presentado por hidatidosis pulmonar y hepática, seguido de tratamiento con albendazol por un período de seis meses.

\section{REPORTE DE CASO}

Paciente de sexo masculino de 54 años de edad natural y procedente de la ciudad de Huancavelica, capital del mismo nombre y localizada a 3676 m de altitud ${ }^{(5)}$.

Acude al hospital por presentar síndrome de obstrucción arterial aguda en el miembro inferior izquierdo, de 6 horas de duración, que es evaluado clínicamente en el Servicio de Emergencia del Hospital Hipólito Unanue (Lima - Perú); se encontró ausencia de pulsos poplíteo y pedio, y leve cianosis distal sin llenado capilar. Se realizaron los exámenes preoperatorios de emergencia cuyos resultados estuvieron dentro de los parámetros normales. Al ser una persona relativamente joven, con un cuadro de inicio brusco, y sin antecedentes de ser portador de fibrilación atrial, se alejaba la posibilidad de embolia arterial por trombo hemático, por lo que el paciente fue sometido a exploración vascular bajo anestesia general a partir de la arteria poplítea izquierda.

Se practicó tromboembolectomía con un catéter de Fogarty $\mathrm{N} .^{\circ} 5$. Con la exploración se removieron múltiples formaciones quísticas de $5 \mathrm{~mm}$ de diámetro y algunas membranas que obstruían la luz de la arteria, además de algunos coágulos, con ello se lograron recuperar los pulsos pedio y poplíteo, y una mejoría de la irrigación sanguínea distal (Figura 1).

Tres días después, se registra un nuevo episodio obstructivo en el mismo territorio vascular por lo que se realizó el mismo procedimiento exploratorio de donde se obtuvo, nuevamente, múltiples membranas correspondientes a quiste hidatídico. Al día siguiente, el paciente fallece súbitamente. Durante su internamiento se realizaron exámenes radiológicos que muestran imágenes compatibles con formaciones quísticas proyectadas a nivel de la imagen cardiaca. En la tomografía axial computada se evidenció reducción del campo pulmonar izquierdo, tractos fibrosos, engrosamiento pleural y obliteración de los senos costo y cardiofrénico derechos, en relación a antecedente quirúrgico de remoción operatoria de un quiste hidatídico pulmonar y hepático realizado 8 años antes (Figura 2).

Se realizó la necropsia el día del fallecimiento y se encontró quistes hidatídicos de diversos tamaños en el parénquima pulmonar, el pericardio, el miocardio, las
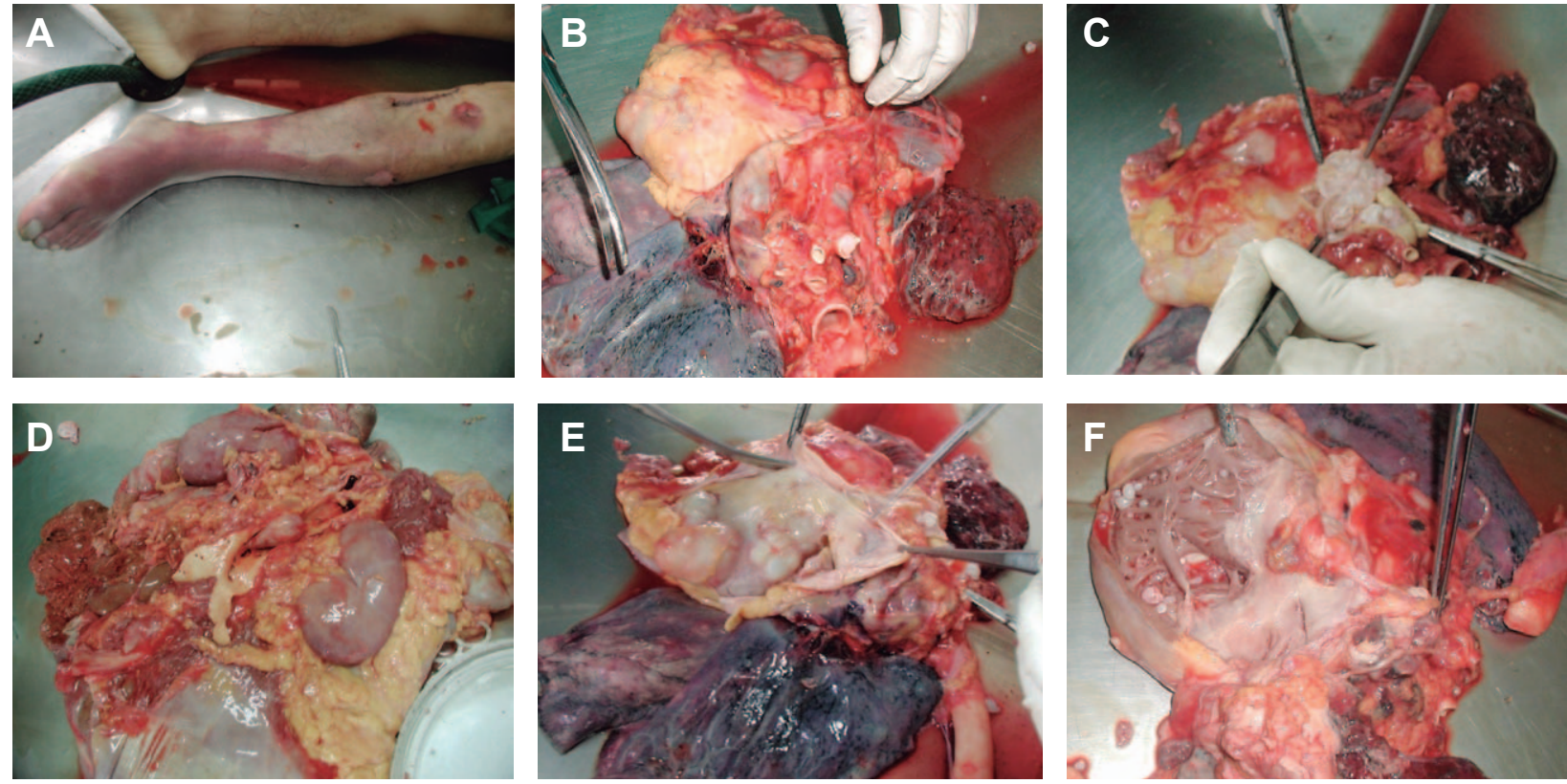

Figura 1. A. Miembro inferior izquierdo que fue explorado con catéter de Fogarty por presentar embolia. B. Desembocadura de las venas pulmonares en la aurícula izquierda. En la luz de las venas pulmonares se observa quistes hidatídicos que las obstruyen. C. Apertura de la aurícula izquierda en donde se observan múltiples quistes hidatídicos adheridos a su pared. D. Se observa quiste hidatídico adherido a la pared en la luz de la arteria aorta abdominal antes de su bifurcación. E. Abierto el pericardio se observan múltiples quistes pericárdicos y miocárdicos. F. En la cavidad ventricular se observa multiples quistes entre las trabéculas. 

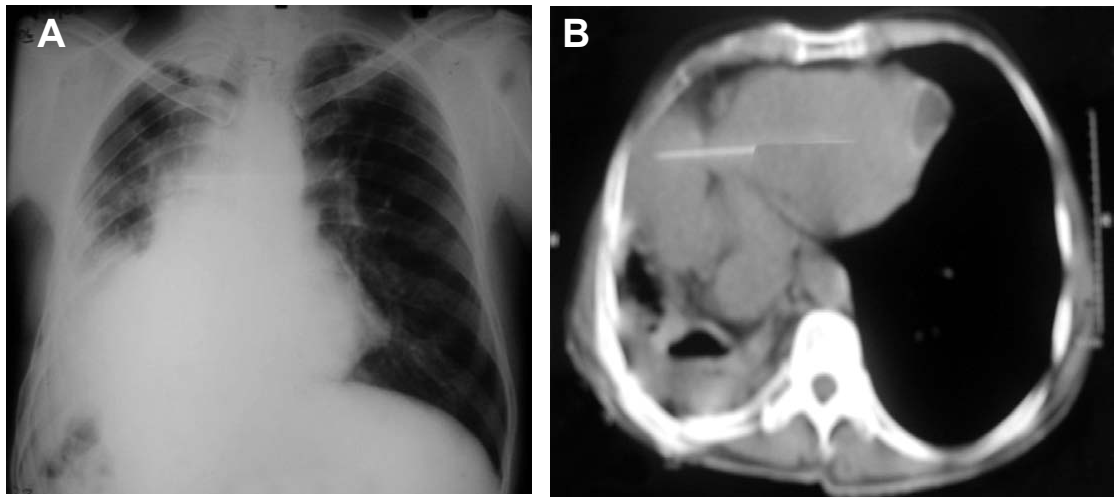

Figura 2. A. Rx de tórax PA se observa reducción evidente del campo pulmonar derecho como secuela de una intervención quirúrgica por hidatidosis hepático pulmonar 8 años antes. En la silueta cardiaca se observa la presencia de una imagen añadida en el borde cardiaco izquierdo que corresponde a quiste hidatídico.

B. TAC se observa formaciones quísticas proyectadas sobre la imagen cardiaca.

cámaras auricular y ventricular izquierda, la arteria aorta abdominal, los riñones y el hígado. La aurícula izquierda se encontró casi completamente ocupada por múltiples quistes de varios tamaños, algunos adheridos fuertemente a la pared auricular y obstruyendo ostensiblemente la desembocadura de las venas pulmonares (Figura 3).

No se examinó el cerebro debido a que los familiares no autorizaron la apertura craneal. Los especímenes de necropsia fueron procesados en el Departamento de Patología Clínica y Anatomía Patológica de Hospital Nacional Hipólito Unanue.

\section{DISCUSIÓN}

La hidatidosis es un problema común en zonas rurales del mundo. Después de la ingestión de los huevos, estos se distribuyen en el cuerpo para formar quistes en los distintos tejidos. Las ubicaciones más comunes de los quistes hidatídicos son el hígado y el pulmón, su frecuencia oscila entre $60-70 \%$ a nivel hepático y entre $15-25 \%$ a nivel pulmonar. Dentro de las localizaciones menos frecuentes encontramos: bazo, riñón, páncreas, mesenterio y corazón, donde esta última es una de las más raras $(0,02-2 \%)$, estado el ventrículo izquierdo
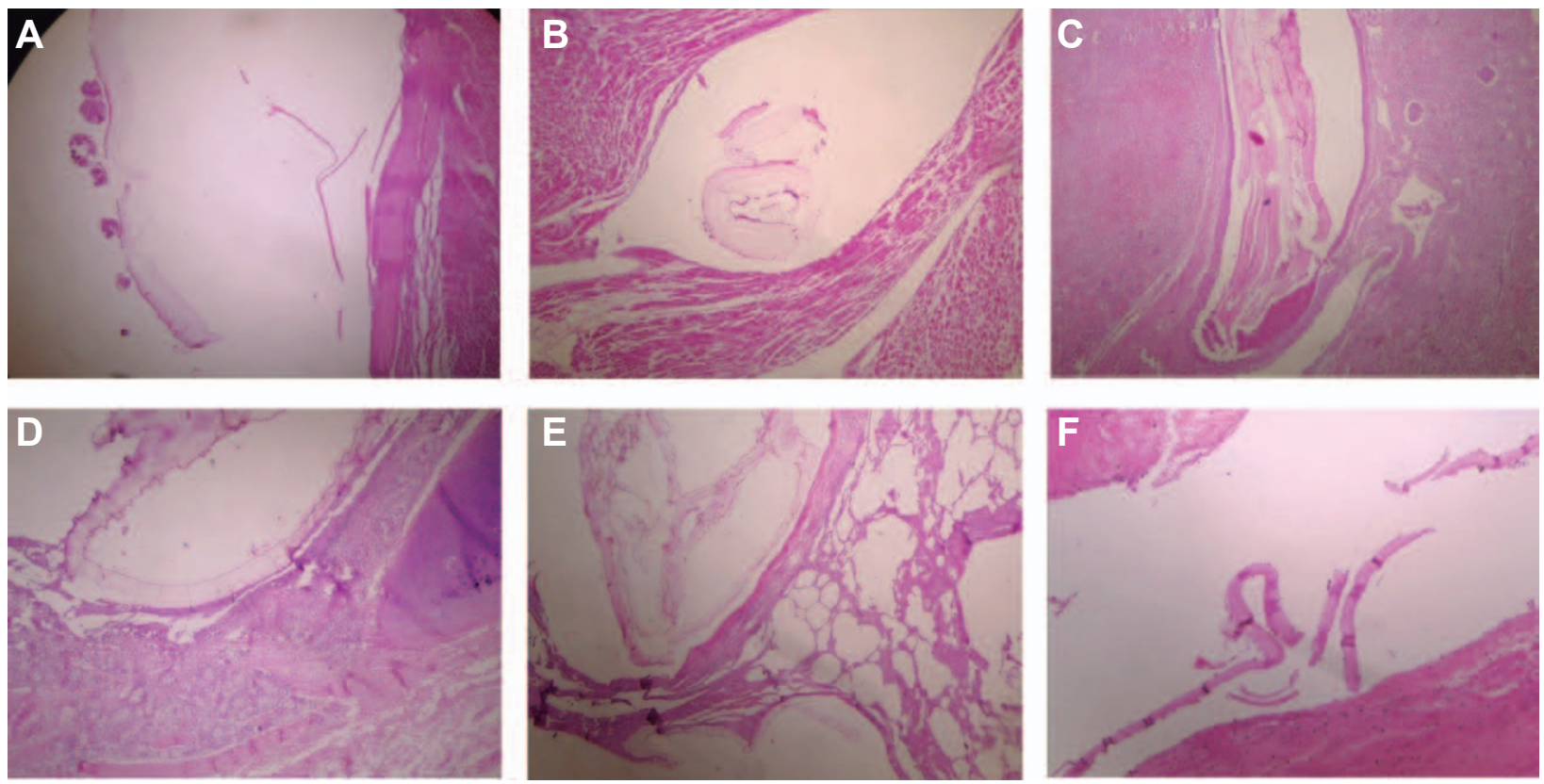

Figura 3. A. Corte de la aurícula izquierda, se observa quiste hidatídico con su capa germinativa, al lado derecho se identifica la adventicia y el músculo cardiaco. (H\&E 100x). B. Corte a nivel del septum ventricular, se observa membranas anhistas de quiste hidatídico dentro del músculo cardiaco, se reconocen fibras cardiacas. (H\&E 100x). C. Corte de riñón, a nivel intraparenquimal se observa un sistema de cavidades en donde se reconocen membranas anhistas de quistes hidatídicos plegadas entre sí. Hacia el margen derecho se observan ovillos glomerulares. No se evidencia respuesta inflamatoria. (H\&E 125x). D. Corte de hilio pulmonar, en la luz del bronquio principal se observa membranas anhistas de quiste hidatídico, además, hiperplasia y gran actividad mucosecretora de las glándulas mucosas. No se observa respuesta inflamatoria (H\&E 100x). E. Corte de pulmón, a nivel intraparenquimal se observa sistema de cavidades ocupadas por quistes hidatídicos, además áreas de enfisema y colapso hacia la derecha. No hay respuesta inflamatoria (H\&E 100x). F. Corte a nivel de coagulo hemático procedente de arteria aorta en donde se observa membranas anhistas de quiste hidatídico (H\&E 100x). 
afectado e, la mayoría de los casos (50-60\%). Además, esta infección no tiene signos y síntomas específicos y menos aún en las localizaciones inusuales, lo cual constituye un reto diagnostico ${ }^{(6,7)}$.

Se han identificado dos mecanismos que utiliza el parásito para combatir la respuesta inmune del hospedero $y$, a su vez, favorecer la cronicidad y persistencia de la infección: a) "escape pasivo", en el cual el parásito se desarrolla dentro del quiste para evitar los efectos perjudiciales de la respuesta inmune; y b) "inmunomodulación", mediante el cual el parásito interacciona activamente con el sistema inmune del hospedero ${ }^{(8)}$.

Se ha visto que la respuesta Th1 está relacionada con resistencia de enfermedad en contraste con citoquinas Th2 que están asociadas a susceptibilidad y cronicidad. Además, se ha evidenciado que los pacientes que responden al tratamiento antiparasitario presentan un predominio de niveles de citoquinas Th1 ${ }^{(9)}$.

El antígeno $\mathrm{B}(\mathrm{AgB})$ del parásito ha sido estudiado ampliamente por tener el rol más importante dentro del mecanismo de inmunomodulación, alterando tanto la inmunidad innata como adquirida del hospedero ${ }^{(10)}$. Además, ha sido demostrado que el AgB debilita la respuesta inflamatoria y dirige el balance Th1/Th2 hacia Th2 ${ }^{(11,12)}$. El AgB utiliza dos estrategias para interferir en la función de las células dendríticas del hospedero. Interfiere en la diferenciación del monocito a célula dendrítica inmadura y modula la maduración de células dendríticas centinelas cebándolas para polarizar los linfocitos a células Th2 ${ }^{(11)}$.

Con relación a la oclusión arterial, son poco los casos que se han reportado, específicamente de la arteria femoral de presentación súbita y diseminación sistémica. Un reporte publicado en el año 1969, menciona el manejo exitoso del embolismo mediante la embolectomía, a pesar de que el paciente ya había tenido otros episodios de quistes hidatídicos abdominales diseminados, no fue una presentación severa y el manejo quirúrgico fue exitoso ${ }^{(13)}$
En general cuando se presenta un caso agudo o crónico de obstrucción arterial, no se sospecha de quistes hidatídicos sino de aterosclerosis; el diagnóstico se realiza durante la operación y el manejo consiste en retirar los quistes posibles con las consiguiente normalización de las características del miembro inferior y los pulsos periféricos, luego de ello se añade el tratamiento médico con albendazol y mebendazol, para prevenir una recurrencia ${ }^{(14)}$

Se ha reportado un caso en el que el embolismo arterial fue la localización primaria, lo cual es extremadamente raro (14). Así mismo, se han presentado casos de embolismo arterial con episodio previo de hidatidosis, generalmente en hígado y pulmón o a nivel del mediastino; pero no como el presentado en este reporte, cuyos lugares de diseminación son diversos, incluyendo pulmón, hígado, riñón, cavidades y paredes del corazón, y arteria aorta ${ }^{(13)}$.

En el presente caso, probablemente, el inicio de la diseminación fue en la aurícula izquierda ya que en dicha cámara se encontró una gran cantidad de quistes hidatídicos en diferentes grados de maduración. Este hallazgo y su conexión con el ventrículo izquierdo sugieren un probable punto de origen de la diseminación sistémica de los quistes.

Entre las complicaciones causadas por quistes hidatídicos si la invasión es vascular incluyen shock anafiláctico, hemorragia, embolia sistémica y oclusión arterial. Siendo el shock anafiláctico una de las causas de muerte súbita por hidatidosis diseminada ${ }^{(15-18)}$. Se puede suponer que la embolia sistémica también llegó al sistema nervioso central comprometiendo gravemente al paciente. Esto no pudo ser corroborado debido a que los familiares no autorizaron explorar esta región.

Contribuciones de autoría: JS participó en en la concepción y diseño del estudio, redacción y revisión crítica del artículo y aprobación de su versión final. GA contribuyó con el reporte y presentación inicial del caso. GLC contribuyó con el estudio anatomo-patológico de las piezas operatorias. JCA, ECP, PBC y PTL aportaron haciendo una revisión exhaustiva de la bibliografía. Todos los autores revisaron de forma crítica la versión final del artículo. 


\section{REFERENCIAS BIBLIOGRÁFICAS}

1. Giri S, Parija SC. A review on diagnostic and preventive aspects of cystic echinococcosis and human cysticercosis. Trop Parasitol. 2012 Jul;2(2):99-108. doi: 10.4103/22295070.105174 .

2. Documet Perea A. Recopilacion de datos sobre hidatidosis y cisticercosis humana en hospitales de Lima, e hidatidosis animal en camales de Lima [Internet]. Lima: 2002 [citado el 4 de febrero del 2014]. Disponible en: http://www.bvsde.paho.org/texcom/ CD045364/hidatidosis.pdf

3. Irabedra P, Salvatella R. The Southern Cone Sub-Regional Project on Cystic Echinococcosis Control and Surveillance. Rev Peru Med Exp Salud Publica. 2010 Oct-Dec;27(4):598-603.

4. Moro PL, Budke CM, Schantz PM, Vasquez J, Santivañez SJ, Villavicencio J. Economic impact of cystic echinococcosis in peru. PLoS Negl Trop Dis. 2011 May;5(5):e1179. doi: 10.1371/journal.pntd.0001179.

5. Perú, Ministerio de Salud, Direccion Regional de Salud. Analisis de Situacion de Salud Huancavelica [Internet]. Huancavelica: MINSA; 2009 [citado el 4 de febrero del 2014]. Disponible en: http://www.bvsde, paho.org/documentosdigitales/bvsde/ texcom/ASIS-regiones/Huancavelica/ Huancavelica2009.pdf

6. Yildirim M, Erkan N, Vardar E. Hydatid cysts with unusual localizations: diagnostic and treatment dilemmas for surgeons. Ann Trop Med Parasitol. 2006 Mar;100(2):137-42.
7. Kıreși DA, Karabacakoğlu A, Odev K, Karaköse S. Uncommon locations of hydatid cysts. Acta Radiol. 2003 Nov;44(6):622-36.

8. Zhang W, Li J, Mcmanus DP. Concepts in immunology and diagnosis of hydatid disease. Clin Microbiol Rev. 2003 Jan;16(1):18-36.

9. Zhang W, Wen $\mathrm{H}, \mathrm{Li} \mathrm{J}$, Lin R, McManus DP. Immunology and immunodiagnosis of cystic echinococcosis: an update. Clin Dev Immunol. 2012;2012:101895. doi: $10.1155 / 2012 / 101895$.

10. Siracusano A, Teggi A, Ortona E. Human cysticechinococcosis: old problems and new perspectives. Interdiscip Perspect Infect Dis. 2009;2009:474368. doi: $10.1155 / 2009 / 474368$.

11. Riganò R, Buttari B, Profumo E, Ortona E, Delunardo F, Margutti P, et al. Echinococcus granulosus antigen B impairs human dendritic cell differentiation and polarizes immature dendritic cell maturation towards a Th2 cell response. Infect Immun. 2007 Apr;75(4):1667-78.

12. Riganò R, Profumo E, Bruschi F, Carulli G, Azzarà A, Ioppolo S, et al. Modulation of human immune response by Echinococcus granulosus antigen $B$ and its possible role in evading host defenses. Infect Immun. 2001 Jan;69(1):288-96.

13. Christeas N, Balas P, Chadjiyiannakis E. Embolism of the femoral artery by an echinococcus cyst successfully treated by embolectomy. Am J Surg. 1968 May;115(5):673-4.
14. Necdet Öz, Cengiz Türkay, Ilhan Gölbaşi, Levent Dertsiz, Alpay Sarper, Abid Demircan. Primary Vascular Echinococcosis: An Uncommon Cause of Chronic Iliofemoral Arterial Occlusion. Tex Heart Inst J. 2000;27(2):20911.

15. Byard RW. An analysis of possible mechanisms of unexpected death occurring in hydatid disease (echinococcosis). J Forensic Sci. 2009 Jul;54(4):919-22. doi: 10.1111/j.15564029.2009.01065.x.

16. Infanger M, Kossmehl P, Grimm D. Surgical and medical management of rare echinococcosis of the extremities: pre- and post-operative long-term chemotherapy. Scand J Infect.Dis. 2005;37(11-12):954-7.

17. Concha F, Maguiña C, Seas C. Disseminated intra-abdominal hydatidosis. Am J Trop Med Hyg. 2013 Sep;89(3):401-2. doi: 10.4269/ ajtmh.13-0083.

18. Goel MM, Verma N, Sagar M. Disseminated intra-abdominal hydatidosis causing acute intestinal obstruction-a rare presentation. BMJ Case Rep. 2010 Oct 4;2010. pii: bcr0620103066. doi: 10.1136/bcr.06.2010.3066.

Correspondecia: Jose Somocurcio

Dirección: Calle Rosa Perez Liendo 132. San Miguel. Lima 32. Perú.

Correo:jotagese@hotmail.com

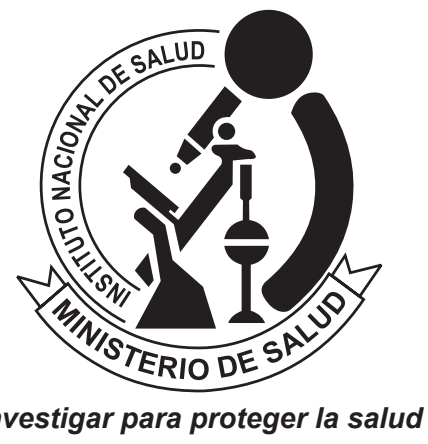

REVISTA PERUANA DE MEDICINA EXPERIMENTAL Y SALUD PÚBLICA CUMPLIENDO SUS METAS Y PROYECTÁNDOSE AL FUTURO 